\title{
Assigning protein function from domain-function associations using DomFun
}

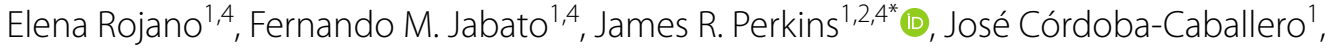 \\ Federico García-Criado ${ }^{1}$, lan Sillitoe ${ }^{3}$, Christine Orengo ${ }^{3}$, Juan A. G. Ranea ${ }^{1,2,4 \dagger}$ and Pedro Seoane-Zonjic ${ }^{1,2,4 \dagger}$
}

\author{
*Correspondence: \\ jimrperkins@gmail.com \\ ${ }^{\dagger}$ J. A. G. Ranea and P. Seoane- \\ Zonjic have contributed \\ equally to this article \\ ${ }^{2}$ CIBER of Rare Diseases, \\ Av. Monforte de Lemos, \\ 3-5. Pabellon 11. Planta 0, \\ 28029 Madrid, Spain \\ Full list of author information \\ is available at the end of the \\ article
}

\begin{abstract}
Background: Protein function prediction remains a key challenge. Domain composition affects protein function. Here we present DomFun, a Ruby gem that uses associations between protein domains and functions, calculated using multiple indices based on tripartite network analysis. These domain-function associations are combined at the protein level, to generate protein-function predictions.

Results: We analysed 16 tripartite networks connecting homologous superfamily and FunFam domains from CATH-Gene3D with functional annotations from the three Gene Ontology (GO) sub-ontologies, KEGG, and Reactome. We validated the results using the CAFA 3 benchmark platform for GO annotation, finding that out of the multiple association metrics and domain datasets tested, Simpson index for FunFam domainfunction associations combined with Stouffer's method leads to the best performance in almost all scenarios. We also found that using FunFams led to better performance than superfamilies, and better results were found for $\mathrm{GO}$ molecular function compared to $\mathrm{GO}$ biological process terms. DomFun performed as well as the highest-performing method in certain CAFA 3 evaluation procedures in terms of $F_{\max }$ and $S_{\min }$ We also implemented our own benchmark procedure, Pathway Prediction Performance (PPP), which can be used to validate function prediction for additional annotations sources, such as KEGG and Reactome. Using PPP, we found similar results to those found with CAFA 3 for GO, moreover we found good performance for the other annotation sources. As with CAFA 3, Simpson index with Stouffer's method led to the top performance in almost all scenarios.
\end{abstract}

Conclusions: DomFun shows competitive performance with other methods evaluated in CAFA 3 when predicting proteins function with $\mathrm{GO}$, although results vary depending on the evaluation procedure. Through our own benchmark procedure, PPP, we have shown it can also make accurate predictions for KEGG and Reactome. It performs best when using FunFams, combining Simpson index derived domain-function associations using Stouffer's method. The tool has been implemented so that it can be easily adapted to incorporate other protein features, such as domain data from other sources, amino acid k-mers and motifs. The DomFun Ruby gem is available from https://rubygems.org/gems/DomFun. Code maintained at https://github.com/Elena author(s) and the source, provide a link to the Creative Commons licence, and indicate if changes were made. The images or other third party material in this article are included in the article's Creative Commons licence, unless indicated otherwise in a credit line to the material. If material is not included in the article's Creative Commons licence and your intended use is not permitted by statutory regulation or exceeds the permitted use, you will need to obtain permission directly from the copyright holder. To view a copy of this licence, visit http:// creativecommons.org/licenses/by/4.0/. The Creative Commons Public Domain Dedication waiver (http://creativecommons.org/publi cdomain/zero/1.0/) applies to the data made available in this article, unless otherwise stated in a credit line to the data. 
Rojano/DomFun. Validation procedure scripts can be found at https://github.com/ ElenaRojano/DomFun_project.

Keywords: Function prediction, CATH, DomFun, Protein domains, CAFA

\section{Background}

Determining protein function is one of the major goals of bioinformatics. A key factor influencing the role of a given protein is its domain composition [1, 2]. Although domains can have distinct functions when examined individually, their combination within a given protein is what gives rise to its overall role in cellular processes [3]. As such, we must first understand individual domains and then investigate how they contribute to protein function. Approaches like dcGO use information from resources such as the Gene Ontology (GO) [4, 5], to statistically infer domain annotation [6].

Various features are important for function prediction, including sequence homology and conserved structure, which are used to classify protein domains by resources such CATH-Gene3D [7, 8], which uses a hierarchical classification system with the most specific group being the homologous superfamily [9].

Within the homologous superfamilies, domains can be further sub-divided into functional families (FunFams), based on shared patterns of sequence conservation [10]. Such domain families have been used by algorithms such as FunFHMMer to predict function at the protein level [9]. Furthermore, FunFam domain information has been used to predict protein functional sites using machine learning [11].

In recent years, we have developed tools to analyse and extract information from network-based data structures $[12,13]$. We used them to find associations between pathological phenotypes and genomic mutations [14-17], and predict the genes involved in the development of rare diseases [18]. Nevertheless, without adequate functional knowledge of the proteins encoded by these genes, we cannot fully understand the underlying mechanisms leading to disease.

Here, we present DomFun, a framework that uses associations between protein domains and functional annotation to predict protein function (Fig. 1).

These associations are calculated by exploiting tripartite networks connecting domains and functional groups via proteins [19], using GO terms and pathways from KEGG [20] and Reactome [21]. For a given protein, DomFun obtains its constituent domains and their functional associations. The association scores are then combined to predict functional annotation.

We validated our method using the prediction benchmark of the third version of the Critical Assessment of Functional Annotation challenge (CAFA 3) [22]. This evaluation method is widely used to evaluate protein function prediction methods. We focussed on $\mathrm{GO}$ annotation for proteins from multiple organisms in the three GO sub-ontologies: molecular function (GOMF), biological process (GOBP) and cellular component (GOCC). We have also developed and applied our own benchmarking protocol, named Pathway Prediction Performance (PPP).

DomFun can be used to predict protein function for multiple organisms for which protein domain and functional annotation information is available. Although we used CATH-Gene3D annotation in this work, other features can be used. It can be downloaded from https://rubygems.org/gems/DomFun. Ruby code is available from https:// 


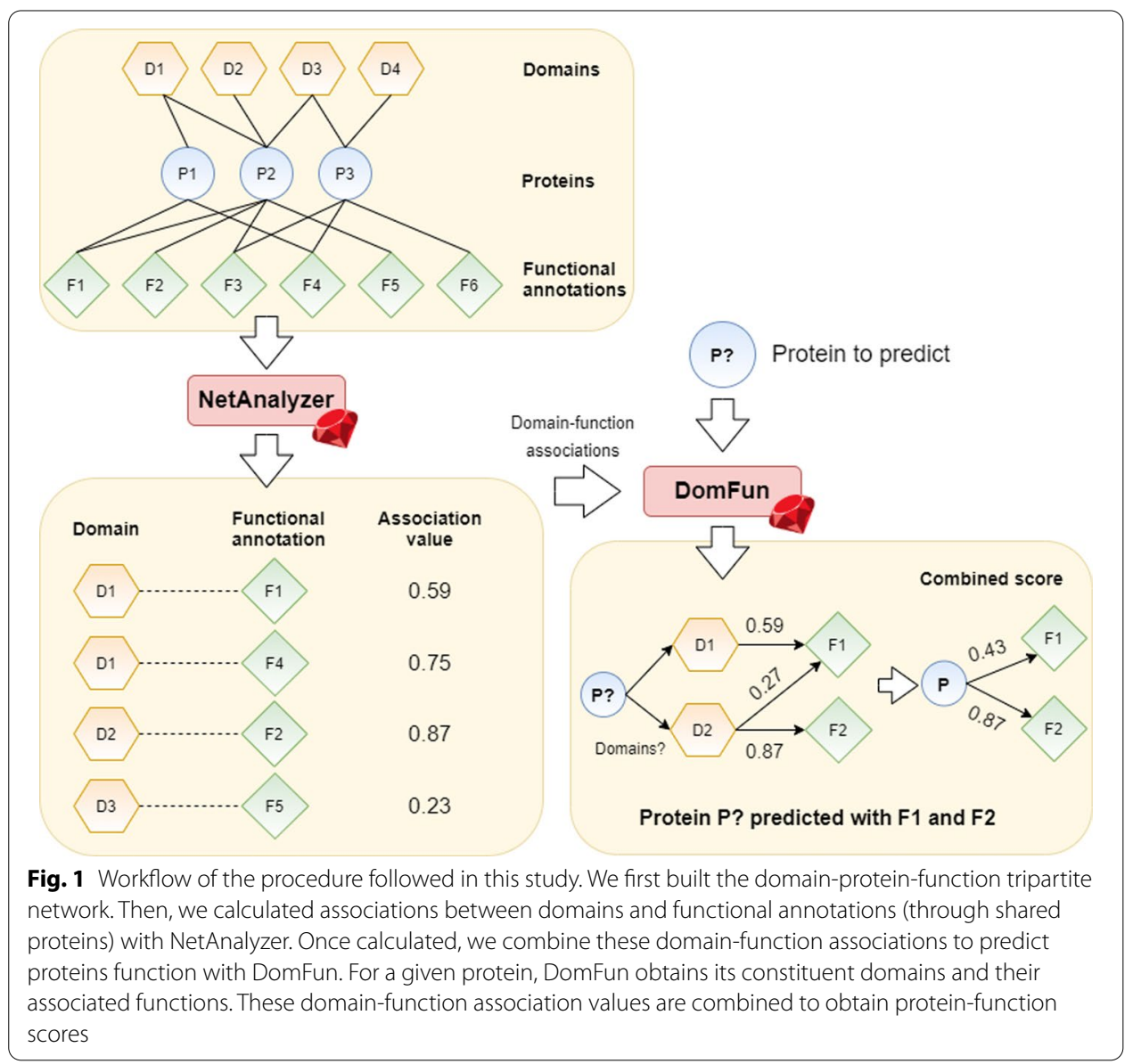

github.com/ElenaRojano/DomFun and the workflow from https:/github.com/Elena Rojano/DomFun_project. Technical information to install and use DomFun is provided in these repositories.

\section{Implementation}

We have developed and implemented DomFun, a tool to predict function for a given protein based on associations between its constituent domains and functions, obtained from various annotation-databases. Associations are calculated using a tripartite network comprised of domains, proteins and functional annotation. The DomFun algorithm works in the following manner: Based on a training dataset, protein-domain and protein-function annotation data are combined to produce a tripartite network of 3 layers: domains-proteins-functions. This tripartite network is analysed using the NetAnalyzer software [12]. NetAnalyzer take as input a multipartite network and calculates associations between different layers within this network. In the context of this work, the layers are: domains, proteins and functions, and the associations are calculated between the domains and functions layers, bases on the connections via proteins. The output is therefore a list of pairs of domains and functions, with corresponding association values. Lists, with their corresponding association values, were calculated for 4 different association indices, mathematical details of which are described in the next section. 
Then, given a testing protein, DomFun predicts function for this protein by obtaining all of its annotated domains and searching for their associated functions in the list generated by NetAnalyzer. The scores for these associated functions are then combined using the methods whose mathematical formulae are described below, to obtain overall scores, which represent the predicted functions for the protein.

An overview of the architecture of the software implementation is described in Fig. 1.

\section{Protein, domain and annotation data sources}

To build the domain-protein-function tripartite networks used to calculate the domainfunction associations with DomFun, two types of relations were combined: proteinfunction and protein-domain.

The workflow followed to perform the different steps explained next and data download links are included in a GitHub repository at https://github.com/ElenaRojano/DomFun_workflow.

\section{Protein-function annotation}

We used two datasets to establish protein-function relations.

The first dataset was based on the functional annotation included in the third Critical Assessment of Functional Annotation challenge (CAFA 3) [22]. We used 66,841 protein identifiers available for the CAFA 3 training set (35,086 annotated in GOMF, 50,813 in GOBP and 49,328 in GOCC) to construct the protein-annotation layer for six tripartite networks. This information was downloaded from CAFA 3 repository at https://www. biofunctionprediction.org/cafa-targets/CAFA3_training_data.tgz.

The second dataset was downloaded from UniProt (release 2021_02). We obtained 23,391,902 proteins both manually curated (Swiss-Prot) and computationally inferred (TrEMBL) for multiple species. We also downloaded their annotations in GOMF, GOBP, GOCC, KEGG and Reactome. We discarded proteins with GO annotation tagged as Inferred from Electronic Annotation (IEA) to ensure high-quality annotations for our study. We also discarded all protein tagged as fragments and protein fusions. We used this information to construct the protein-annotation layer for ten different tripartite networks. After these filters, we were left with 4,283,876 annotated proteins. Please note that the same protein may have annotation in any of the GO sub-ontologies, KEGG or Reactome.

\section{Protein-domain annotation}

To establish protein-domain relations, we used protein domains classified into homologous superfamilies and FunFams from the protein structure classification database CATH-Gene3D [8]. Superfamily classification is performed by grouping sequences likely to have an evolutionary relationship [9]. FunFams are a sub-classification of superfamilies based on shared patterns of sequence conservation related to function determining residues [23]. For this analysis, we used the CATH-Gene3D release v4_3_0, including 1,307,795 proteins from 1705 species, 4245 different superfamilies and 171,425 FunFams. 


\section{Tripartite network construction and association index calculation}

We obtained multiple sets of protein-function and protein-domain relations. In total we built 16 networks connecting domains to functional annotation via shared proteins, one for each combination of protein-domain and protein-function datasets.

These domain-protein-function networks were analysed with NetAnalyzer, a tool that calculates associations in multipartite networks [12]. We employed this tool to calculate domain-function associations using the Jaccard similarity index (eq. 1), Simpson index (eq. 2), Pearson correlation coefficient (PCC) (eq. 3) and the hypergeometric index (HyI) (eq. 4), as described in [24].

$$
\begin{aligned}
& \operatorname{Jaccard}(D, F)=\frac{\left|N_{p}(D) \cap N_{p}(F)\right|}{\left|N_{p}(D) \cup N_{p}(F)\right|} \\
& \operatorname{Simpson}(D, F)=\frac{\left|N_{p}(D) \cap N_{p}(F)\right|}{\min \left(\left|N_{p}(D)\right|,\left|N_{p}(F)\right|\right)} \\
& P C C(D, F)=\frac{\left|N_{p}(D) \cap N_{p}(F)\right| \cdot n_{T}-\left|N_{p}(D) \cdot N_{p}(F)\right|}{\sqrt{\left|N_{p}(D)\right| \cdot\left|N_{p}(F)\right| \cdot\left(n_{T}-\left|N_{p}(D)\right|\right) \cdot\left(n_{T}-\left|N_{p}(F)\right|\right)}} \\
& H y I(D, F)=-\log \sum_{i=\left|N_{p}(D) \cap N_{p}(F)\right|} \frac{\left(\begin{array}{c}
\left|N_{p}(D)\right| \\
i
\end{array}\right) \cdot\left(\begin{array}{c}
n_{T}-\left|N_{p}(D)\right| \\
\left|N_{p}(F)\right|-i
\end{array}\right)}{\left(\begin{array}{c}
n_{T} \\
\left|N_{p}(F)\right|
\end{array}\right)}
\end{aligned}
$$

where $N_{p}(D)$ and $N_{p}(F)$ are the set of protein nodes connected to a given domain node $\mathrm{D}$ and a function node $\mathrm{F}$, respectively, and $n_{T}$ is the total number of protein nodes in the network.

\section{Protein function prediction based on domain-function associations}

The domain-function associations calculated using the above methods were used to predict protein function using DomFun. First, for a given protein, associated with a specific UniProt identifier, DomFun searches for its constituent domains within CATH-Gene3D. If domains are found, it then searches for any functions associated with them. If a protein contains multiple domains associated with the same functional annotation (Fig. 1), DomFun integrates the association values into a single combined score. This leads to a list of possible functions for the protein, ranked based on the strength of the domain-function associations, as described in the next section.

By calculating this score for all functions associated with at least one domain for a given protein, we obtain a vector of scores, which represents the predicted functions for the protein.

DomFun outputs a table of predicted functions for each protein, containing the UniProt identifier, the domains for that protein classified according to $\mathrm{CATH}$ Gene3D superfamilies or FunFams, the predicted functions (GOMF, GOBP, GOCC, KEGG and Reactome) and the combined score for each putative protein-function association. 


\section{Combining domain-function association values}

As mentioned above, if a protein contains multiple domains with the same function, these scores are combined into a single value. In the case of $\mathrm{HyI}$ values, these must be transformed into $P$-values by calculating their antilogarithm (base $=10)$, which represents the probability of having an equal or greater number of interactions between a pair of nodes (i.e., proteins connecting domains and functional annotations) than would be expected by chance [24]. To integrate these $P$-values we use the Fisher's combined probability test (eq. 5).

$$
X^{j}=-2 \sum_{i=1}^{k} \ln \left(p_{i}^{j}\right) \sim \chi_{2 k}^{2}
$$

Where $p_{i}^{j}$ is the HyI-derived $P$-value for the number of interactions between a function $j$ and the domain $i$, and $2 k$ represents the degrees of freedom. $k$ represents the total number of domains for each predicted protein. This formula gives the test statistic, from which the combined $P$-value can be derived, based on the $\chi^{2}$ distribution and degrees of freedom.

When combining association values produced using PCC, Jaccard or Simpson index, Stouffer's method was used to obtain, for each of the three metrics, combined association values between proteins and functions (eq. 6) [25]. For this, the association values are first converted to $Z$-scores and then combined using the following formula:

$$
Z^{j}=\frac{\sum_{i=1}^{k} Z_{i}^{j}}{\sqrt{k}} \sim Z
$$

For a given protein, an overall $Z$-score was calculated for each of the functions $j$ associated with at least one domain $i$ within this protein. This was calculated by summing the $Z_{i}^{j}$ scores for the domains in the protein associated with the given function and dividing by the square root of $\mathrm{k}$ - the number of domains in the protein associated with the given function.

The $Z_{i}^{j}$ scores were calculated for each given domain-function association value, by subtracting the mean association value for all domain-function associations, and dividing by the standard deviation.

$$
Z_{i}^{j}=\frac{a_{i}^{j}-\bar{a}}{s}
$$

Where $a$ represents the association values for all calculated domain-function associations; $a_{i}^{j}$ represents the association value for the domain $i$ with a given function $j$; $\bar{a}$ represents the mean association value for all domain-function associations; and $s$ represents the standard deviation for the values in $a$.

\section{DomFun evaluation methods}

We evaluated the ability of DomFun to predict protein function using the CAFA 3 prediction benchmark for GOBP, GOMF and GOCC, using the methodology described in [26] with data available from the CAFA 3 website (https://www.biofunctionprediction. 
org/cafa). We also developed our own validation procedure to evaluate DomFun in terms of predicting function for KEGG and Reactome pathways. We refer to this procedure as Pathway Prediction Performance (PPP). We also used PPP for GOMF, GOBP and GOCC annotations - as similar evaluation values for the three GO sub-ontologies to those found with CAFA 3 would suggest that our benchmark procedure is reliable and lend confidence to the interpretation of the PPP results for KEGG and Reactome.

For this validation, we looked at the maximum value of the harmonic mean $\left(F_{\max }\right)$ of precision and recall (PR), and $S_{\text {min }}$, based on the semantic distance between predictions and known annotations, in line with CAFA 3.

In total, we made eight separate sets of predictions, for the four different association metrics, separately for FunFams and superfamilies. Rather than compare all eight sets against all methods benchmarked within the CAFA 3 results (146 distinct methods), we initially compared the eight sets to each other, to see which performed best across all testing scenarios. The top performing method was then compared against the best performing method from CAFA 3 for each scenario.

\section{CAFA 3 prediction benchmark}

We used data from CAFA 3, a challenge aimed at comparing various methods for predicting protein function, to evaluate the predictive capability of DomFun [22]. In brief, the idea was that competitors would predict annotation for a number of proteins, and then compare their predictions to experimentally determined functional annotation obtained during a given time-period $\left(t_{0}-t_{1}\right)$. As a result, they acquired and published a dataset including a list of the proteins that obtained annotation during this time period, their annotations at the start of the challenge $\left(t_{0}\right)$ and at the end of the challenge $\left(t_{1}\right)$, and a benchmarking procedure with scripts to implement it.

The CAFA 3 dataset includes various sub-divisions to evaluate the prediction methods, as explained in [22, 26], including two modes of evaluation: full and partial, and two types of annotation: no knowledge and limited knowledge. Full evaluation penalizes models if they cannot predict for all GO sub-ontology terms; partial mode evaluates without this penalization.

With respect to the different proteins in the benchmark testing set, no knowledge proteins are those with no experimentally verified annotation in any of the three GO subontologies at time $t_{0}$, but that accumulate at least one verified GO term between $t_{0}$ and $t_{1}$. Limited knowledge includes proteins with annotation in at least one GO sub-ontology, but not in all three at $\mathrm{t}_{0}[22,26]$. We used different combinations to see with which one of our methods performed best in different scenarios.

We used the CAFA protein-centric evaluation mode. It calculates maximum F-measure $F_{\text {max }}$, using PR values for the proteins for which predictions could be made, to ascertain the performance of the predictive method. It also calculates the minimum semantic distance $\left(S_{\text {min }}\right)$ between two GO terms (one from prediction and the other from the ground truth, i.e. CAFA 3). In addition, it calculates coverage, defined in this work as the fraction of benchmark proteins for which DomFun could make predictions. Formulae of these evaluation metrics are described in [26].

The CAFA 3 benchmark files include 3089 proteins. We consider this set as our testing set. We predicted GOMF, GOBP and GOCC for 2483 proteins from this set. As 
occurred with the training proteins set, we lost 606 testing proteins as they had no CATH domains. This loss affects prediction performance in terms of coverage.

We compared DomFun performance against the two baseline models, Naïve and BLAST. We generated both models following instructions provided by CAFA authors [22]. We also compared the performance of our methods with the top scoring methods from CAFA 3, in terms of both $F_{\max }$ and $S_{\min }$, for all three ontologies and all four combinations of evaluation type and mode. Analyses were made using all organisms.

According to CAFA submission rules, prediction scores must be within the $(0.00,1.00]$ range. Therefore, we normalized the combined scores for each protein. In the case of scores calculated using Stouffer's method, as PCC values were within a range of $[-1.00,1.00]$, their absolute values were calculated before they were combined. For Jaccard and Simpson combined scores, normalization was performed as follows: combined scores were transformed to $Z$-scores and any value with absolute value greater than 2 was set to 2 . Finally, the distribution was normalised to the $(0.00,1.00]$ range by dividing each value by the distribution range (4) and adding 0.5 . In the case of scores calculated using Fisher's method, i.e. the combined HyI derived $P$-values, we transformed them into the $(0.00,1.00]$ range by subtracting the $P$-value generated by the method from 1 . To avoid spurious predictions, we removed predictions with a transformed score lower than 0.001 .

To compare the results of the different association/combination methods used by DomFun on the CAFA 3 testing proteins, for both FunFams and superfamilies, we compared their results in terms of $F_{\max }$ and $S_{\min }$ using the Friedman test. If this gave a significant result ( $p \leq 0.05$ ), meaning there is a difference between groups, this was followed by post-hoc pair-wise Dunn's tests to identify a significant difference between the top ranked method and the other methods $(p \leq 0.05)$ [27].

\section{Pathway prediction performance}

In the pathway prediction performance (PPP) validation procedure, we predicted protein function (across all three GO sub-ontologies, KEGG and Reactome) for all proteins that were used to build the domain-protein-function tripartite networks. We compared these predicted associations to the original protein-function associations, and built precision-recall (PR) curves. For this, all predicted annotations that matched the original annotation were considered true positives; all predictions that did not match the original annotation were considered false positives. This additional validation procedure was necessary to evaluate the results obtained using KEGG and Reactome. As we had already validated the $\mathrm{GO}$ annotation using the CAFA 3 benchmarking procedure, we were able to use the PPP procedure to compare the results using KEGG and Reactome to the GO results and put them in the context of the CAFA 3 predictions. PR curves were generated using the ROCR package [28].

\section{Results}

We associated protein domains with functions by applying various metrics to tripartite networks formed by combining protein-domain and protein-function annotation via shared proteins. Functional annotation was obtained from the Gene Ontology molecular 
function (GOMF), biological process (GOBP) and cellular component (GOCC) subontologies and KEGG and Reactome pathways.

The domain-function associations were then used by DomFun for protein function prediction. Two validation procedures were used, one using the training and testing proteins from the CAFA 3 benchmark dataset [22], and another based on all Uniprot proteins (Pathway Prediction Performance, PPP).

\section{CAFA 3 benchmark results}

The CAFA 3 training set contains 98,567 proteins from multiple organisms, of which 50,813 had annotations in GOBP, 35,086 in GOMF and 49,328 in GOCC [22]. We used all proteins from these sets that had CATH-Gene3D annotation to build domain-protein-function tripartite networks for each of the sub-ontologies. In total, there were 41,453 proteins with domain and GOBP annotation, 30,650 with GOMF and 39,144 with GOCC, corresponding to a loss of $18.42 \%, 12.64 \%$ and $20.64 \%$ proteins, respectively, from the training set.

We used the domain-function associations calculated for the CAFA 3 training proteins at $t_{0}$ to predict GO annotation for the 3,089 testing proteins included in CAFA 3, of which 2,483 had domain annotation. Predictions were compared to the annotation obtained for these proteins between $t_{0}$ and $t_{1}$.

We analysed the results to evaluate which combination of domain family, association and integration methods led to the most accurate predictions. This was performed separately for the FunFam and homologous superfamily classifications. We initially compared the results of each method to each other and to the two CAFA 3 baseline methods (BLAST and Naïve) in terms of maximum F-measure $F_{\max }$ (Fig. 2). The exact values are shown in Table 1 for the CAFA 3 limited-knowledge, partial evaluation procedure. Full results for all four CAFA 3 evaluation procedures are shown in Additional file 1: Table S1. In terms of $F_{\max }$, we obtained higher values when using FunFam domains compared to superfamilies for all three GO sub-ontologies. In fact, using superfamily annotation, DomFun performed worse than the baseline methods in most cases. Better results were found in general when predicting both GOMF and GOCC annotation than GOBP; this trend also occurs with the top CAFA 3 methods [22].

Simpson index with Stouffer's method applied to FunFams ranked highest on average amongst all association indices implemented here using NetAnalyzer, for all evaluation procedures across all sub-ontologies, according to both $F_{\max }$ and minimum semantic distance $\left(S_{\min }\right)$. Moreover, for $F_{\max }$ it was the best performing method in all cases, with the exception of the no knowledge, full evaluation (Type 1, Mode 1) comparison for the molecular function sub-ontology (Table 2). Similar results were seen for the $S_{\min }$ output measure (Additional file 2: Table S2).

We compared the results obtained by DomFun to the top results from CAFA 3 for all four evaluation procedure combinations and all three GO sub-ontologies (Table 3). DomFun was the top method using GOMF for the limited knowledge partial evaluation procedure and competitive in several other situations. Similar results were found for $S_{\text {min }}$, with DomFun obtaining a lower score than the top CAFA 3 method for the no knowledge full evaluation procedure and the limited knowledge partial evaluation procedure (Additional file 3: Table S3). For both $F_{\max }$ and $S_{\min }$, DomFun tended to obtain 


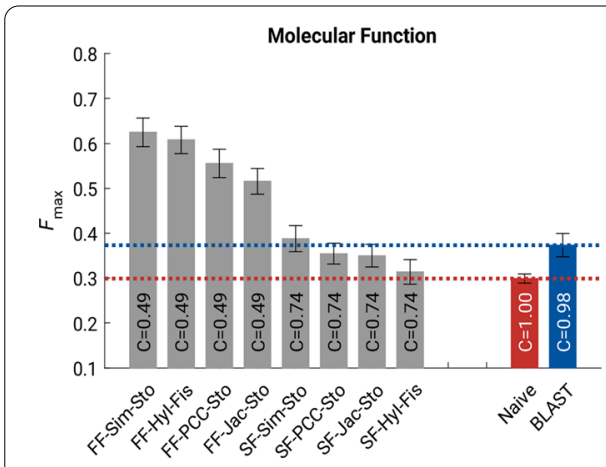

(a) Molecular Function (GOMF)

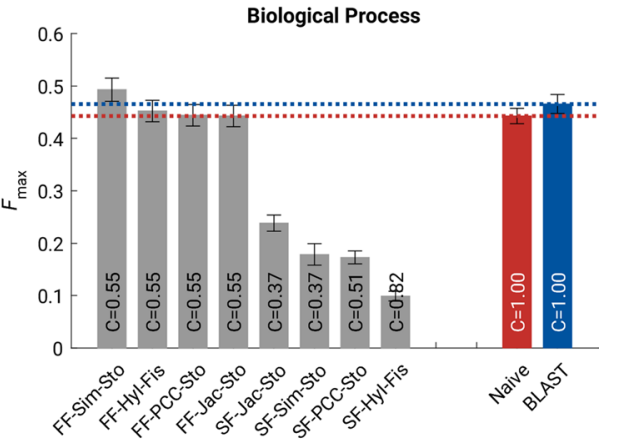

(b) Biological Process (GOBP)

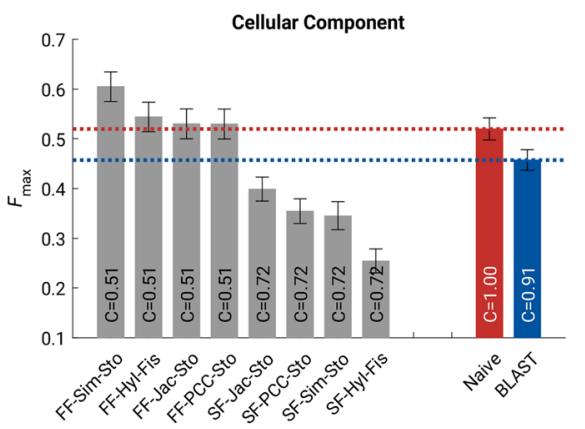

(c) Cellular Component (GOCC)

Fig. 2 Domfun evaluation in terms of maximum F-measure $\left(F_{\max }\right)$ calculation when predicting for Gene Ontology (GO) molecular function (GOMF) (a), biological process (GOBP) (b) and cellular component (c) (GOCC) terms using the CAFA 3 prediction benchmark. The associations between GO terms and protein domains, classified using FunFams (FF) and superfamilies (SF) separately, were calculated using four different association indices: Jaccard (Jac), Simpson (Sim), Pearson correlation coefficient (PCC) and hypergeometric index (Hyl), and combined using either Fisher's method (Fis) or Stouffer's method (Sto). Results for the baseline methods BLAST and Naïve are also included for comparison. Coverage (C) values for each method are included within the bars. The CAFA 3 evaluation procedure was set to partial mode and limited knowledge

Table 1 Maximum F-measure $\left(F_{\max }\right)$ scores obtained with DomFun using the CAFA 3 prediction benchmark

\begin{tabular}{|c|c|c|c|c|c|}
\hline \multirow[t]{2}{*}{ Domain classification } & \multirow[t]{2}{*}{ FA } & \multicolumn{4}{|c|}{ Association + combination methods } \\
\hline & & $\mathrm{Hyl}+$ Fis & PCC+Sto & $\mathrm{Jac}+$ Sto & Sim + Sto \\
\hline \multicolumn{6}{|c|}{$F_{\text {max }}$ values from PR curves (domain-FunFys associations for CAFA 3 target proteins) } \\
\hline \multirow[t]{3}{*}{ FunFams } & GOMF & 0.608 & 0.553 & 0.515 & 0.624 \\
\hline & GOBP & 0.452 & 0.444 & 0.443 & 0.492 \\
\hline & GOCC & 0.542 & 0.529 & 0.529 & 0.602 \\
\hline \multirow[t]{3}{*}{ Superfamilies } & GOMF & 0.314 & 0.347 & 0.350 & 0.384 \\
\hline & GOBP & 0.099 & 0.172 & 0.238 & 0.174 \\
\hline & GOCC & 0.254 & 0.353 & 0.398 & 0.340 \\
\hline
\end{tabular}

The best performing methods for each domain/GO subontology combination are indicated in bold

FA Functional annotation, Hyl hypergeometric index, Sim Simpson index, PCC Pearson correlation coefficient, Jac Jaccard index, Sto Stouffer's combination method, Fis Fisher's combined probability test. CAFA 3 evaluation procedure set to partial mode and limited knowledge 
Rojano et al. BMC Bioinformatics

(2022) 23:43

Page 11 of 19

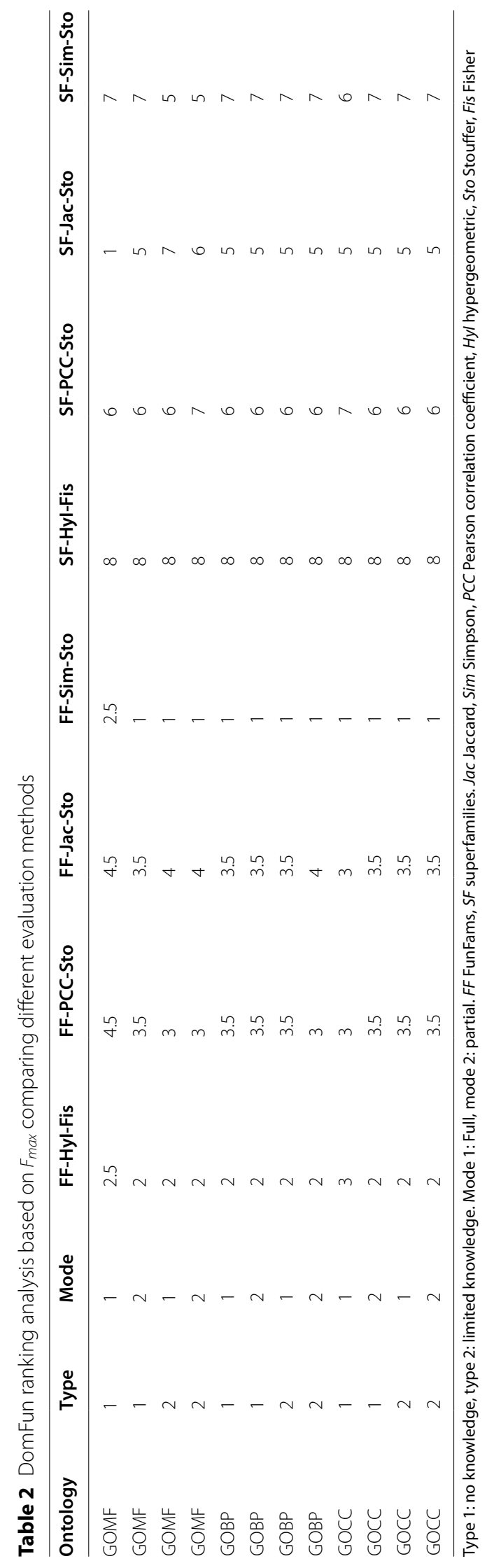


Table $3 F_{\text {max }}$ top values: DomFun (Simpson + Stouffer) vs. CAFA 3 methods

\begin{tabular}{lllllll}
\hline Ontology & Type & Mode & $\begin{array}{l}\text { Top DomFun } \\
F_{\max }\end{array}$ & $\begin{array}{l}\text { DomFun } \\
\text { coverage }\end{array}$ & Top CAFA 3 $F_{\max }$ & $\begin{array}{l}\text { CAFA 3 } \\
\text { coverage }\end{array}$ \\
\hline GOMF & 1 & 1 & 0.357 & 0.71 & 0.618 & 1 \\
GOMF & 1 & 2 & 0.567 & 0.41 & 0.622 & 0.02 \\
GOMF & 2 & 1 & 0.431 & 0.49 & 0.622 & 1 \\
GOMF & 2 & 2 & 0.624 & 0.49 & 0.623 & 0.88 \\
GOBP & 1 & 1 & 0.275 & 0.46 & 0.397 & 1 \\
GOBP & 1 & 2 & 0.402 & 0.46 & 0.418 & 0.62 \\
GOBP & 2 & 1 & 0.37 & 0.55 & 0.598 & 1 \\
GOBP & 2 & 2 & 0.492 & 0.55 & 0.64 & 0.83 \\
GOCC & 1 & 1 & 0.412 & 0.49 & 0.615 & 1 \\
GOCC & 1 & 2 & 0.606 & 0.49 & 0.908 & 1 \\
GOCC & 2 & 1 & 0.422 & 0.51 & 0.615 & 0 \\
GOCC & 2 & 2 & 0.602 & 0.51 & 0.825 &
\end{tabular}

Type 1: no knowledge, type 2: limited knowledge. Mode 1: full evaluation, mode 2: partial evaluation

Table 4 Maximum F-measure $\left(F_{\max }\right)$ scores for precision and recall (PR) curves obtained with DomFun using the Pathway Prediction Performance benchmark procedure

\begin{tabular}{|c|c|c|c|c|c|}
\hline \multirow[t]{2}{*}{ Domains classification } & \multirow[t]{2}{*}{ FA } & \multicolumn{4}{|c|}{ Association + combination methods } \\
\hline & & $\mathrm{Hyl}+$ Fis & PCC + Sto & Jac + Sto & Sim + Sto \\
\hline \multicolumn{6}{|c|}{$F_{\max }$ values from PR curves (domains-FunFys associations from UniProt proteins) } \\
\hline \multirow[t]{5}{*}{ FunFams } & GOMF & 0.779 & 0.749 & 0.749 & 0.850 \\
\hline & GOBP & 0.643 & 0.604 & 0.604 & 0.714 \\
\hline & GOCC & 0.750 & 0.704 & 0.704 & 0.824 \\
\hline & KEGG & 0.730 & 0.730 & 0.730 & 0.822 \\
\hline & Reactome & 0.762 & 0.680 & 0.663 & 0.822 \\
\hline \multirow[t]{5}{*}{ Superfamilies } & GOMF & 0.241 & 0.370 & 0.373 & 0.139 \\
\hline & GOBP & 0.196 & 0.291 & 0.305 & 0.089 \\
\hline & GOCC & 0.266 & 0.221 & 0.217 & 0.129 \\
\hline & KEGG & 0.132 & 0.340 & 0.271 & 0.494 \\
\hline & Reactome & 0.127 & 0.344 & 0.327 & 0.081 \\
\hline
\end{tabular}

The best performing methods for each domain/annotation source combination are indicated in bold

FA Functional annotation, Hyl hypergeometric index, PCC Pearson correlation coefficient, Jac Jaccard index, Sim Simpson index, Sto Stouffer's method, Fis Fisher's method

worse coverage than the best performing CAFA 3 method, although this was not always the case, particularly for $S_{\text {min }}$. All values of $S_{\min }$ and coverage, for all methods, sub-ontologies and validation procedures, are shown in Additional files 4 and 5: Tables S4 and S5.

\section{Pathway prediction performance results}

We further validated DomFun using the PPP validation procedure. Precision and recall (PR) curves for are shown in the Additional files 6 and 7: Figs. S1 and S2. $F_{\max }$ values are shown in Table 4. As with CAFA 3 validation, we observe better AUC-PR values for FunFam predictions than superfamilies.

We compared the $F_{\max }$ values calculated using PPP (Table 4) against those for the CAFA 3 benchmark (Table 1). 
We observed similar $F_{\max }$ values for GOMF, GOCC and GOBP predictions for both CAFA 3 and PPP evaluations using FunFams. These results gives us confidence in the validity of DomFun for predicting KEGG and Reactome pathways. Interestingly, the predictions for these pathways lead to similar $F_{\max }$ values to those calculated for GOMF and GOCC.

The highest $F_{\max }$ values for predictions using FunFams correspond to those calculated using Simpson index with Stouffer's method, in line with the results obtained using the CAFA 3 dataset and lending confidence to the potential use of PPP as a further validation system that can be extended beyond GO to other annotation databases.

With respect to the predictions performed with superfamilies, the $F_{\max }$ values for GOMF, GOBP and GOCC were lower in comparison with FunFams, again suggesting that our methodology works better with FunFams, as also shown in the CAFA 3 results.

\section{Discussion}

We have presented DomFun, a novel approach to predict protein function based on associations between domains and functions. The method is based on the same protein domains classification system used by the FunFHMMer method [9], evaluated in CAFA 3 under the name of Orengo-FunFams [22].

Although both methods are based on similar underlying data, DomFun differs fundamentally from Orengo-FunFams in terms of how it assigns functions to the test proteins. Orengo-FunFams first assigns FunFam domains to a test protein then, for each of these FunFams, obtains corresponding GO term annotations, which are scored based on their frequency among the seed sequences for the given FunFam. Parental terms of these GO terms are also obtained. Finally, the set of all domain-GO terms annotations for the test protein IS considered. This differs markedly from our approach, which first obtains GO association values for all domains in a given protein based on tripartite network analysis, and then combines these values to produce a single score for each predicted proteinannotation association using data-fusion methods.

Like the Orengo-FunFams method, DomFun performs particularly well for the noknowledge partial evaluation and for GOMF. However, it performs less well in full evaluation procedures. The loss of training proteins due to the lack of CATH domains could explain this. As they will not form part of the association network, their information will not be available to make predictions and this could consequently decrease $F_{\max }$ values.

More concretely, we obtained Fmax values of 0.624 (GOMF) and 0.492 (GOBP) for the no knowledge partial evaluation procedure (full details in Table 3); for the OrengoFunFams method these were 0.623 (GOMF) and 0.64 (GOBP). We also performed a comparison between our method and the Orengo-FunFams method using the CAFA 2 training/testing dataset (data not shown), in which the Orengo-FunFams method evaluation showed Fmax values of 0.58 for GOMF, and 0.39 for GOBP when making predictions for Homo sapiens (Figures and 7C and 7L of the CAFA 2 supplementary material [26]). These values were similar to those obtained with DomFun (Table 1) for GOMF calculated with Simpson index with Stouffer's method (0.592) and slightly higher for GOBP calculated with the same method (0.341).

Regarding coverage, we tended to obtain lower values than many of the top-performing CAFA 3 methods, particularly for FunFam predictions, although there were some 
exceptions, as shown in Table 3 and Additional file 3: Table S3. Again, we are currently limited to predicting for proteins for which domain annotation is available. However, the methodology presented here can easily be adapted to incorporate other protein features, such as amino acid k-mers or motifs. To this end have made all code fully available, allowing the user to analyse a tripartite network including any feature of interest. Future work could look into optimizing our system increasing the number of protein domains from other databases, such as Pfam or SCOP, or other protein features.

We obtained better coverage when the associations were calculated using superfamilies than with FunFams, which is not surprising as not all sequences in a superfamily are classified into FunFams. FunFams are only generated for groups of sequences where at least one member has experimental characterisation [23, 29]. As such they can be considered more functionally coherent than superfamilies, which is likely to account for the improved performance.

Simpson index with Stouffer's combination method using FunFams was the best performing of all the methods implemented here for both the CAFA 3 and PPP benchmark, both for $F_{\max }$ and $S_{\min }$. In previous work by Clancy and Hovig, the Simpson index was used to calculate the similarity between pairs of genes mapped to a protein interaction matrix [30]. In their study, the authors explain that the similarity between two genes can vary significantly depending on the number of proteins used for its calculation. To solve this problem they used the Simpson index, which normalises the results of the similarity calculation by the node that has the minimum number of connections (see eq. 2 in the Implementation section) [30].

We have a similar problem, as there can be a large discrepancy in size between the numbers of proteins mapping to each domain and function. We suggest that by using Simpson here, we reduce this problem by normalizing to the smaller of the two.

Notably, there was much more variation in terms of performance for superfamilies. This may also be related to the network degree, this tended to be greater than for FunFams, as superfamilies tend to contain larger numbers of proteins. It should also be made clear that the homologous superfamily classification is based on domains having similar structures, but this does not necessarily mean they will have similar functions.

To validate our results using the CAFA 3 data, we incorporated the original Matlab scripts (https://github.com/yuxjiang/CAFA2) into an automated system that is able to extract testing and training data from the benchmark dataset, build the tripartite networks to calculate associations and combine them to predict function for the testing proteins such that we could perform validation using CAFA3 in a high-throughput manner. These scripts are available from https://github.com/ElenaRojano/DomFun_project and can be used by others to validate their own method, or to validate adaptations of our methods, for example by adding additional protein features to the tripartite network.

The PPP benchmark led to similar $F_{\max }$ values compared to the CAFA 3 benchmark for all three GO sub-ontologies, especially for GOMF and GOCC. As such, we have confidence in the PPP procedure in terms of judging relative performance. Based on these assumptions, it would appear that DomFun has slightly greater accuracy when predicting KEGG and Reactome annotation than GO (Table 4).

The $F_{\max }$ results for GOMF and GOCC tended to be better than for GOBP, in line with CAFA 3. These differences are likely due to the distinct focus of the different 
annotation systems. GOBP terms can be quite varied regarding the different activities of proteins they encompass: these terms refer to biological processes that can involve a large number of distinct molecular activities, enzymatic reactions and regulatory processes. On the other hand, GOMF terms tend to represent more concrete molecular activities. As such, GOBP can be considered harder to predict for [31]. We hypothesize that proteins with similar molecular functions require similar domains to perform their activities, whereas proteins involved the same biological process might utilise a much wider range of domain structures, although this will also depend on the level of specificity of the term within the GO hierarchy.

This is also commented on in the CAFA 2 manuscript, where they argue that predictors return different results depending on the type of ontology used, and that their size (number of elements), depth (maximum degree of specificity) and branching factor (number of total connections between nodes) can affect the results [26].

As mentioned above, the PPP benchmark showed better results for KEGG than GO. These resources include well-curated representations of specific metabolic pathways with shared catalytic activities performed by multiple proteins, which may explain these findings. Further work could investigate methodology to select the more specific GO terms using semantic similarity measures [32].

\section{Conclusion}

Results for both CAFA 3 and PPP validation show that, of the 8 prediction frameworks implemented here, combining the four association metrics with the two protein domain annotation types (superfamilies and FunFam), the best combination for making domain-function predictions with DomFun is the Simpson index with Stouffer's method using FunFams.

We have shown that protein domain associations based on network analysis can be useful for predicting protein function for multiple species, showing comparable performance when predicting GO annotation to other methods based on structural domain-based in the CAFA 3 challenge. We have also presented a novel validation system for protein function prediction that shows similar results to the CAFA 3 benchmark, but can also be extended to use KEGG and Reactome annotation.

DomFun has been implemented in such a way that other protein features, such as different domain annotation classifications can also be integrated. Future work of DomFun should focus on improving domain-function associations using additional protein-features for protein annotation, more domain annotations and predicting for a greater range of functional systems.

\section{Availability and requirements}

Project name: DomFun Project home page: https://github.com/ElenaRojano/DomFun project Operating system(s): Unix-like systems Programming language: Ruby, Matlab Other requirements: CAFA 3 benchmarking system used to compare methods License: GNU GPL Any restrictions to use by non-academics: None. 


\section{Abbreviations}

AUC-PR: Area under the curve-precision and recall; CAFA: Critical Assessment of Function Annotation; $F_{\text {max }}$ : Maximum F-measure; FunFam: Functional family; Hyl: Hypergeometric index; GO: Gene Ontology; GOBP: Gene Ontology biological process; GOCC: Gene Ontology cellular component; GOMF: Gene Ontology molecular function; KEGG: Kyoto Encyclopedia of Genes and Genomes; PCC: Pearson correlation coefficient; PR: Precision and recall; $S_{\text {min }}$ : Minimum semantic difference.

\section{Supplementary Information}

The online version contains supplementary material available at https://doi.org/10.1186/s12859-022-04565-6.

Additional file 1. Table S1: $F_{\max }$ values for all DomFun methods and all combinations of evaluation scenarios and ontologies, compared to the highest equivalent value from CAFA 3 and the baseline methods. Type 1: no knowledge, type 2: limited knowledge. Mode 1: Full, mode 2: partial. FF: FunFams, SF: superfamilies. Jac: Jaccard, Sim: Simpson, PCC: Pearson correlation coe_cient, Hyl: hypergeometric. Sto: Stou_er, Fis: Fisher.

Additional file 2. Table S2

Additional file 3. Table S3: Domfun $S_{\min } T_{0 p}$ v. CAFA $S_{\min } T o p$ (top means lowest score). Type 1: no knowledge, type 2: limited knowledge. Mode 1: Full, mode 2: partial.

Additional file 4. Table S4: Coverage values for all DomFun methods and all combinations of evaluation scenarios and ontologies, compared to the highest equivalent value from CAFA 3 and the baseline methods. Type 1: no knowledge, type 2: limited knowledge. Mode 1: Full, mode 2: partial. FF: FunFams, SF: superfamilies. Jac: Jaccard, Sim: Simpson, PCC: Pearson correlation coefficient, Hyl: hypergeometric. Sto: Stouffer, Fis: Fisher.

Additional file 5. Table S5: $S_{\min }$ values for all DomFun methods and all combinations of evaluation scenarios and ontologies, compared to the highest equivalent value from CAFA 3 and the baseline methods. Type 1: no knowledge, type 2: limited knowledge. Mode 1: Full, mode 2: partial. FF: FunFams, SF: superfamilies. Jac: Jaccard, Sim: Simpson, PCC: Pearson correlation coefficient, Hyl: hypergeometric. Sto: Stouffer, Fis: Fisher.

Additional file 6. Fig. S1: Precision and recall curves to ascertain DomFun accuracy using domain-function associations with FunFams (PPP). funfamsPPP.pdf Prediction results for Gene Ontology (GO) molecular functions (GOMF, red curves), biological process (GOBP, blue curves), GOCC (green curves), KEGG (orange curves) and Reactome pathways (black curves) are shown. These curves compare DomFun results using associations between FunFam domains and functions calculated with (a) Jaccard index, (b) Pearson Correlation Coefficient (PCC), (c) hypergeometric index (Hyl) and (d) Simpson index. The area under the precision-recall curve (AUC-PR) for each comparison is also shown.

Additional file 7. Fig. S2: Precision and recall curves to ascertain DomFun accuracy using domain-function associations with superfamilies (PPP). superfamilyPPP.pdf Prediction results for Gene Ontology (GO) molecular functions (GOMF, red curves), biological process (GOBP, blue curves), GOCC (green curves), KEGG (orange curves) and Reactome pathways (black curves) are shown. These curves compare DomFun results using associations between superfamily domains and functions calculated with (a) Jaccard index, (b) Pearson Correlation Coefficient (PCC), (c) hypergeometric index ( $\mathrm{Hyl}$ ) and (d) Simpson index. The area under the precision-recall curve (AUC-PR) for each comparison is also shown.

\section{Acknowledgements}

The authors would like to thank the team of Prof. Christine Orengo for their technical support during the realization of this work. ER thanks Mr. Andrew Perkins and Mrs. Catherine Perkins for their support during the accomplishment of this work throughout her stay in London. The authors thank the Supercomputing and Bioinnovation Center (SCBI) of the University of Malaga for their provision of computational resources and technical support (http://www.scbi.uma.es/site).

\section{Authors' contributions}

ER and JRP wrote the manuscript and co-developed with PSZ and FMJ the tools that were necessary to perform the analysis. FGC was crucial for the description and formulation of the methods. ER, JRP, JCC, JAR and PSZ analysed the biological content of the results. IS and CO provided the datasets necessary to perform this analysis. All authors revised the manuscript critically for important intellectual content and have read and approved the final manuscript. All authors read and approved the final manuscript.

\section{Funding}

This work was supported by the Spanish Ministry of Science, Innovation and Universities with European Regional Development Fund [SAF2016-78041-C2-1-R, PID2019-108096RB-C21, PID2019-105010RB-100], the Andalusian Government with European Regional Development Fund [UMA18-FEDERJA-102, PAIDI 2020:PY20-00372], the Ramón Areces foundation, which funds project for the investigation of rare disease (National call for research on life and material sciences, XIX edition) and the University of Malaga (Ayudas del I Plan Propio). The CIBERER is an initiative from the Institute of Health Carlos III. JRP holds a research grant from the Andalusian Government (Fundacion Progreso y Salud) [PI-0075-2017]. ER is a postdoctoral researcher from the Plan de Formacion de Personal Investigador (FPI) supported by the Andalusian Government. The funding bodies did not play any role in the study and collection, analysis, and interpretation of data and in writing the manuscript. 


\section{Availability of data and materials}

The source code and datasets used in this research can be downloaded from https://rubygems.org/gems/DomFun, https://github.com/ElenaRojano/DomFun and https://github.com/ElenaRojano/DomFun_project.

\section{Declarations}

Ethics approval and consent to participate

Not applicable.

\section{Consent to publish}

Not applicable.

\section{Competing interests}

The authors declare that they have no competing interests.

\section{Author details}

${ }^{1}$ Department of Molecular Biology and Biochemistry, University of Malaga, Bulevar Louis Pasteur, 31, 29010 Malaga, Spain. ${ }^{2}$ CIBER of Rare Diseases, Av. Monforte de Lemos, 3-5. Pabellon 11. Planta 0, 28029 Madrid, Spain. ${ }^{3}$ Department of Structural and Molecular Biology, University College London, Gower Street, London WC1E 6BT, UK. ${ }^{4}$ Institute of Biomedical Research in Malaga (IBIMA), Dr. Miguel Díaz Recio, 28, 29010 Malaga, Spain.

Received: 5 October 2020 Accepted: 5 January 2022

Published online: 15 January 2022

\section{References}

1. Rey FA. One protein, many functions. Nature. 2010;468:773-5. https://doi.org/10.1038/468773a.

2. Ponting CP, Russell RR. The natural history of protein domains. Annu Rev Biophys Biomol Struct. 2002;31(1):4571. https://doi.org/10.1146/annurev.biophys.31.082901.134314.

3. Forslund K, Sonnhammer ELL. Predicting protein function from domain content. Bioinformatics. 2008;24(15):1681-7. https://doi.org/10.1093/bioinformatics/btn312.

4. Ashburner M, Ball CA, Blake JA, Botstein D, Butler H, Cherry JM, Davis AP, Dolinski K, Dwight SS, Eppig JT, Harris MA, Hill DP, Issel-Tarver L, Kasarskis A, Lewis S, Matese JC, Richardson JE, Ringwald M, Rubin GM, Sherlock G. Gene ontology: tool for the unification of biology. 2000. https://doi.org/10.1038/75556.

5. Carbon S, Douglass E, Dunn N, Good B, Harris NL, Lewis SE, Mungall CJ, Basu S, Chisholm RL, Dodson RJ, Hartline E, Fey P, Thomas PD, Albou LP, Ebert D, Kesling MJ, Mi H, Muruganujan A, Huang X, Poudel S, Mushayahama T, Hu JC, LaBonte SA, Siegele DA, Antonazzo G, Attrill H, Brown NH, Fexova S, Garapati P, Jones TEM, Marygold SJ, Millburn GH, Rey AJ, Trovisco V, Dos Santos G, Emmert DB, Falls K, Zhou P, Goodman JL, Strelets VB, Thurmond J, Courtot M, Osumi DS, Parkinson H, Roncaglia P, Acencio ML, Kuiper M, Lreid A, Logie C, Lovering RC, Huntley RP, Denny P, Campbell NH, Kramarz B, Acquaah V, Ahmad SH, Chen H, Rawson JH, Chibucos MC, Giglio M, Nadendla S, Tauber R, Duesbury MJ, Del NT, Meldal BHM, Perfetto L, Porras P, Orchard S, Shrivastava A, Xie Z, Chang HY, Finn RD, Mitchell AL, Rawlings ND, Richardson L, Sangrador-Vegas A, Blake JA, Christie KR, Dolan ME, Drabkin HJ, Hill DP, Ni L, Sitnikov D, Harris MA, Oliver SG, Rutherford K, Wood V, Hayles J, Bahler J, Lock A, Bolton ER, De Pons J, Dwinell M, Hayman GT, Laulederkind SJF, Shimoyama M, Tutaj M, Wang SJ, D'Eustachio P, Matthews L, Balhoff JP, Aleksander SA, Binkley G, Dunn BL, Cherry JM, Engel SR, Gondwe F, Karra K, MacPherson KA, Miyasato SR, Nash RS, Ng PC, Sheppard TK, Shrivatsav Vp A, Simison M, Skrzypek MS, Weng S, Wong ED, Feuermann M, Gaudet P, Bakker E, Berardini TZ, Reiser L, Subramaniam S, Huala E, Arighi C, Auchincloss A, Axelsen K, Argoud GP, Bateman A, Bely B, Blatter MC, Boutet E, Breuza L, Bridge A, Britto R, Bye-A-Jee H, Casals-Casas C, Coudert E, Estreicher A, Famiglietti L, Garmiri P, Georghiou G, Gos A, Gruaz-Gumowski N, Hatton-Ellis E, Hinz U, Hulo C, Ignatchenko A, Jungo F, Keller G, Laiho K, Lemercier P, Lieberherr D, Lussi Y, Mac-Dougall A, Magrane M, Martin MJ, Masson P, Natale DA, Hyka NN, Pedruzzi I, Pichler K, Poux S, Rivoire C, Rodriguez-Lopez M, Sawford T, Speretta E, Shypitsyna A, Stutz A, Sundaram S, Tognolli M, Tyagi N, Warner K, Zaru R, Wu C, Chan J, Cho J, Gao S, Grove C, Harrison MC, Howe K, Lee R, Mendel J, Muller HM, Raciti D, Van Auken K, Berriman M, Stein L, Sternberg PW, Howe D, Toro S, Westerfield M. The Gene Ontology Resource: 20 years and still GOing strong. Nucleic Acids Res (2019). https://doi.org/10.1093/nar/gky1055

6. Fang H, Gough J. DcGO: database of domain-centric ontologies on functions, phenotypes, diseases and more. Nucleic Acids Res. 2013. https://doi.org/10.1093/nar/gks1080.

7. Orengo CA, Michie AD, Jones S, Jones DT, Swindells MB, Thornton JM. CATH—a hierarchic classification of protein domain structures. Structure. 1997;5(8):1093-108. https://doi.org/10.1016/s0969-2126(97)00260-8.

8. Sillitoe I, Lewis TE, Cuff A, Das S, Ashford P, Dawson NL, Furnham N, Laskowski RA, Lee D, Lees JG, Lehtinen S, Studer RA, Thornton J, Orengo CA. CATH: comprehensive structural and functional annotations for genome sequences. Nucleic Acids Res. 2015;43(Database issue):376-81. https://doi.org/10.1093/nar/gku947.

9. Das S, Sillitoe I, Lee D, Lees JG, Dawson NL, Ward J, Orengo CA. CATH FunFHMMer web server: protein functional annotations using functional family assignments. Nucleic Acids Res. 2015;43(Web server issue):148-53. https:// doi.org/10.1093/nar/gkv488.

10. Dawson NL, Lewis TE, Das S, Lees JG, Lee D, Ashford P, Orengo CA, Sillitoe I. CATH: an expanded resource to predict protein function through structure and sequence. Nucleic Acids Res. 2017;45(D1):289-95. https://doi. org/10.1093/nar/gkw1098. 
11. Scheibenreif L, Littmann M, Orengo C, Rost B. FunFam protein families improve residue level molecular function prediction. BMC Bioinform. 2019;20(400):66. https://doi.org/10.1186/s12859-019-2988-X.

12. Rojano E, Seoane P, Bueno-Amoros A, Perkins JR, Garcia-Ranea JA. Revealing the relationship between human genome regions and pathological phenotypes through network analysis. In: Rojas I, Ortuño F (eds) International conference on bioinformatics and biomedical engineering: 5th international work conference, IWBBIO 2017, Granada, Spain: Springer; 2017. p. 197-207.

13. Jabato FM, Rojano E, Perkins JR, Ranea JAG, Seoane-Zonjic P. Kernel based approaches to identify hidden connections in gene networks using NetAnalyzer. In: Lecture notes in computer science (including subseries lecture notes in artificial intelligence and lecture notes in bioinformatics) (2020). https://doi.org/10.1007/978-3-03045385-5_68.

14. Reyes-Palomares A, Bueno A, Rodríguez-López R, Medina MÁ, Sánchez-Jiménez F, Corpas M, Ranea JAGG. Systematic identification of phenotypically enriched loci using a patient network of genomic disorders. BMC Genomics. 2016;17(1):232. https://doi.org/10.1186/s12864-016-2569-6.

15. Bueno A, Rodríguez-López R, Reyes-Palomares A, Rojano E, Corpas M, Nevado J, Lapunzina P, Sánchez-Jiménez F, Ranea JAGG. Phenotype-loci associations in networks of patients with rare disorders: application to assist in the diagnosis of novel clinical cases. Eur J Hum Genet. 2018;26:1451-61. https://doi.org/10.1038/s41431-018-0139-x.

16. Jabato FM, Seoane P, Perkins JR, Rojano E, García Moreno A, Chagoyen M, Pazos F, Ranea JAG. Systematic identification of genetic systems associated with phenotypes in patients with rare genomic copy number variations. Human Genet. 2020;6:66. https://doi.org/10.1007/s00439-020-02214-7.

17. Díaz-Santiago E, Jabato FM, Rojano E, Seoane P, Pazos F, Perkins JR, Ranea JAG. Phenotype-genotype comorbidity analysis of patients with rare disorders provides insight into their pathological and molecular bases. PLOS Genet. 2020;16(10):1009054.

18. Rojano E, Seoane-Zonjic P, Jabato FM, Perkins JR, Ranea JAG. Comprehensive analysis of patients with undiagnosed genetic diseases using the Patient Exploration Tools Suite (PETS). In: Lecture notes in computer science (including subseries lecture notes in artificial intelligence and lecture notes in bioinformatics). 2020. https://doi. org/10.1007/978-3-030-45385-5_69

19. Rojano E, Perkins JR, Sillitoe I, Orengo C, García Ranea JA, Seoane P. Associating protein domains with biological functions: a tripartite network approach. In: Lecture notes in computer science (including subseries lecture notes in artificial intelligence and lecture notes in bioinformatics). 2019. p. 155-64. https://doi.org/10.1007/9783-030-17935-9_15.

20. Ogata H, Goto S, Sato K, Fujibuchi W, Bono H, Kanehisa M. KEGG: Kyoto encyclopedia of genes and genomes. 1999. arXiv:1011.1669v3. https://doi.org/10.1093/nar/27.1.29.

21. Fabregat A, Jupe S, Matthews L, Sidiropoulos K, Gillespie M, Garapati P, Haw R, Jassal B, Korninger F, May B, Milacic M, Roca CD, Rothfels K, Sevilla C, Shamovsky V, Shorser S, Varusai T, Viteri G, Weiser J, Wu G, Stein L, Hermjakob H, D'Eustachio P. The Reactome pathway knowledgebase. Nucleic Acids Res. 2018;44(Database issue):481-7. https://doi.org/10.1093/nar/gkx1132.

22. Zhou N, Jiang Y, Bergquist TR, Lee AJ, Kacsoh BZ, Crocker AW, Lewis KA, Georghiou G, Nguyen HN, Hamid MN, Davis L, Dogan T, Atalay V, Rifaioglu AS, Dalkiran A, Cetin-Atalay R, Zhang C, Hurto RL, Freddolino PL, Zhang Y, Bhat P, Supek F, Fernández JM., Gemovic B, Perovic VR, Davidović RS, Sumonja N, Veljkovic N, Asgari E, Mofrad MR, Profiti G, Savojardo C, Martelli PL, Casadio R, Boecker F, Kahanda I, Thurlby N, McHardy AC, Renaux A, Saidi R, Gough J, Freitas AA, Antczak M, Fabris F, Wass MN, Hou J, Cheng J, Hou J, Wang Z, Romero AE, Paccanaro A, Yang H, Goldberg T, Zhao C, Holm L, Törönen P, Medlar AJ, Zosa E, Borukhov I, Novikov I, Wilkins A, Lichtarge O, Chi P-H, Tseng W-C, Linial M, Rose PW, Dessimoz C, Vidulin V, Dzeroski S, Sillitoe I, Das S, Lees JG, Jones DT, Wan C, Cozzetto D, Fa R, Torres M, Vesztrocy AW, Rodriguez JM, Tress ML, Frasca M, Notaro M, Grossi G, Petrini A, Re M, Valentini G, Mesiti M, Roche DB, Reeb J, Ritchie DW, Aridhi S, Alborzi SZ, Devignes M-D, Koo DCE, Bonneau R, Gligorijević V, Barot M, Fang H, Toppo S, Lavezzo E, Falda M, Berselli M, Tosatto SC, Carraro M, Piovesan D, Rehman HU, Mao Q, Zhang S, Vucetic S, Black GS, Jo D, Larsen DJ, Omdahl AR, Sagers LW, Suh E, Dayton JB, McGuffin LJ, Brackenridge DA, Babbitt PC, Yunes JM, Fontana P, Zhang F, Zhu S, You R, Zhang Z, Dai S, Yao S, Tian W, Cao R, Chandler C, Amezola M, Johnson D, Chang J-M, Liao W-H, Liu Y-W, Pascarelli S, Frank Y, Hoehndorf R, Kulmanov M, Boudellioua I, Politano G, Carlo SD, Benso A, Hakala K, Ginter F, Mehryary F, Kaewphan S, Björne J, Moen H, Tolvanen MEE, Salakoski T, Kihara D, Jain A, Šmuc T, Altenhoff A, Ben-Hur A, Rost B, Brenner SE, Orengo CA, Jeffery CJ, Bosco G, Hogan DA, Martin MJ, O'Donovan C, Mooney SD, Greene CS, Radivojac P, Friedberg I. The CAFA challenge reports improved protein function prediction and new functional annotations for hundreds of genes through experimental screens. Genome Biol. 2019. https://doi.org/10.1101/653105.

23. Rentzsch R, Orengo CA. Protein function prediction using domain families. BMC Bioinform. 2013. https://doi.org/ 10.1186/1471-2105-14-S3-S5.

24. Bass JIF, Diallo A, Nelson J, Soto JM, Myers CL, Walhout AJM. Using networks to measure similarity between genes: association index selection. Nat Methods. 2013;10(12):1169-76. https://doi.org/10.1038/nmeth.2728.

25. Stouffer SA, Suchman EA, Devinney LC, Star SA., Williams RM, Jr. The American Soldier: adjustment during army life. Studies in Social Psychology in World War II, vol 1; 1949.

26. Jiang Y, Oron TR, Clark WT, Bankapur AR, D'Andrea D, Lepore R, Funk CS, Kahanda I, Verspoor KM, Ben-Hur A, Koo DCE, Penfold-Brown D, Shasha D, Youngs N, Bonneau R, Lin A, Sahraeian SME, Martelli PL, Profiti G, Casadio R, Cao R, Zhong Z, Cheng J, Altenhoff A, Skunca N, Dessimoz C, Dogan T, Hakala K, Kaewphan S, Mehryary F, Salakoski T, Ginter F, Fang H, Smithers B, Oates M, Gough J, Törönen P, Koskinen P, Holm L, Chen CT, Hsu WL, Bryson K, Cozzetto D, Minneci F, Jones DT, Chapman S, Bkc D, Khan IK, Kihara D, Ofer D, Rappoport N, Stern A, Cibrian-Uhalte E, Denny P, Foulger RE, Hieta R, Legge D, Lovering RC, Magrane M, Melidoni AN, Mutowo-Meullenet P, Pichler K, Shypitsyna A, Li B, Zakeri P, ElShal S, Tranchevent LC, Das S, Dawson NL, Lee D, Lees JG, Sillitoe I, Bhat P, Nepusz T, Romero AE, Sasidharan R, Yang H, Paccanaro A, Gillis J, Sedeño-Cortés AE, Pavlidis P, Feng S, Cejuela JM, Goldberg T, Hamp T, Richter L, Salamov A, Gabaldon T, Marcet-Houben M, Supek F, Gong Q, Ning W, Zhou Y, Tian W, Falda M, Fontana P, Lavezzo E, Toppo S, Ferrari C, Giollo M, Piovesan D, Tosatto SCE, del Pozo A, Fernández JM, Maietta P, Valencia A, Tress ML, Benso A, Di Carlo S, Politano G, Savino A, Rehman HU, Re M, Mesiti 
M, Valentini G, Bargsten JW, van Dijk ADJ, Gemovic B, Glisic S, Perovic V, Veljkovic V, Veljkovic N, Almeida-e-Silva DC, Vencio RZN, Sharan M, Vogel J, Kansakar L, Zhang S, Vucetic S, Wang Z, Sternberg MJE, Wass MN, Huntley RP, Martin MJ, O'Donovan C, Robinson PN, Moreau Y, Tramontano A, Babbitt PC, Brenner SE, Linial M, Orengo CA, Rost B, Greene CS, Mooney SD, Friedberg I, Radivojac P. An expanded evaluation of protein function prediction methods shows an improvement in accuracy. Genome Biol. 2016;17(1):184. https://doi.org/10.1186/s13059-0161037-6.1601.00891.

27. Dunn OJ. Multiple comparisons using rank sums. Technometrics. 1964;6(3):241. https://doi.org/10.2307/12660 41.

28. Sing T, Sander O, Beerenwinkel N, Lengauer T. ROCR: visualizing classifier performance in R. Bioinformatics. 2005;21(20):3940-1. https://doi.org/10.1093/bioinformatics/bti623.

29. Das S, Lee D, Sillitoe I, Dawson NL, Lees JG, Orengo CA. Functional classification of CATH superfamilies: a domain-based approach for protein function annotation. Bioinformatics. 2015. https://doi.org/10.1093/bioin formatics/btv398.

30. Clancy T, Hovig E. Differential protein network analysis of the immune cell lineage. BioMed Res Int. 2014;6:66. https://doi.org/10.1155/2014/363408.

31. Jaramillo-Garzón JA, Gallardo-Chacón JJ, Castellanos-Domínguez CG, Perera-Lluna A. Predictability of gene ontology slim-terms from primary structure information in Embryophyta plant proteins. BMC Bioinform. 2013;14(68):66. https://doi.org/10.1186/1471-2105-14-68.

32. Zhao Y, Fu G, Wang J, Guo M, Yu G. Gene function prediction based on Gene Ontology hierarchy preserving hashing. Genomics. 2019;111(3):334-42. https://doi.org/10.1016/j.ygeno.2018.02.008.

\section{Publisher's Note}

Springer Nature remains neutral with regard to jurisdictional claims in published maps and institutional affiliations.

- fast, convenient online submission

- thorough peer review by experienced researchers in your field

- rapid publication on acceptance

- support for research data, including large and complex data types

- gold Open Access which fosters wider collaboration and increased citations

- maximum visibility for your research: over $100 \mathrm{M}$ website views per year

At BMC, research is always in progress.

Learn more biomedcentral.com/submissions 